\title{
THE CLASS OF DISTRIBUTIONS ASSOCIATED WITH THE GENERALIZED POLLACZEK-KHINCHINE FORMULA
}

\author{
OFFER KELLA,* The Hebrew University of Jerusalem
}

\begin{abstract}
The goal is to identify the class of distributions to which the distribution of the maximum of a Lévy process with no negative jumps and negative mean (equivalently, the stationary distribution of the reflected process) belongs. An explicit new distributional identity is obtained for the case where the Lévy process is an independent sum of a Brownian motion and a general subordinator (nondecreasing Lévy process) in terms of a geometrically distributed sum of independent random variables. This generalizes both the distributional form of the standard Pollaczek-Khinchine formula for the stationary workload distribution in the $\mathrm{M} / \mathrm{G} / 1$ queue and the exponential stationary distribution of a reflected Brownian motion.

Keywords: Generalized Pollaczek-Khinchine formula; Lévy process with no negative jumps; spectrally positive Lévy process; reflected Lévy process; supremum of a Lévy process
\end{abstract}

2010 Mathematics Subject Classification: Primary 60G51; 60K25

\section{Introduction and preliminaries}

Let $X=\left\{X_{t} \mid t \geq 0\right\}$ be a Lévy process with no negative jumps. It is standard knowledge that in this case $\mathrm{Ee}^{-\alpha X_{t}}=\mathrm{e}^{\varphi(\alpha) t}$ is finite for all $\alpha \geq 0$ and that

$$
\varphi(\alpha)=b \alpha+\frac{\sigma^{2}}{2} \alpha^{2}+\int_{(0, \infty)}\left(\mathrm{e}^{-\alpha x}-1+\alpha x \mathbf{1}_{\{x \leq 1\}}\right) v(\mathrm{~d} x),
$$

where $b$ is real, $\sigma$ is nonnegative, and $v$ is a measure satisfying

$$
\int_{(0, \infty)}\left(x^{2} \wedge 1\right) v(\mathrm{~d} x)<\infty
$$

where $a \wedge b=\min (a, b)$.

It is also well known that $-\mathrm{E} X_{t} / t=\varphi^{\prime}(0)=b-\int_{(1, \infty)} x v(\mathrm{~d} x)$ and, in particular, $\mathrm{E} X_{t}$ is well defined and can be either finite or $+\infty$ but never $-\infty$. In particular, if $\mathrm{E} X_{t}<0$ (equivalently, $\left.\varphi^{\prime}(0)>0\right)$ then $\int_{(1, \infty)} x v(\mathrm{~d} x)<b$, so that in particular $b>0$ and $\int_{(1, \infty)} x v(\mathrm{~d} x)<\infty$. Therefore, in this case $\varphi$ has the form

$$
\varphi(\alpha)=\mu \alpha+\frac{\sigma^{2}}{2} \alpha^{2}+\int_{(0, \infty)}\left(\mathrm{e}^{-\alpha x}-1+\alpha x\right) \nu(\mathrm{d} x),
$$

where $\mu=\varphi^{\prime}(0)=b-\int_{(1, \infty)} x v(\mathrm{~d} x)>0$.

Received 6 December 2011; revision received 12 April 2012.

* Postal address: Department of Statistics, The Hebrew University of Jerusalem, Mount Scopus, Jerusalem 91905, Israel. Email address: offer.kella@huji.ac.il

Supported in part by grant 434/09 from the Israel Science Foundation and the Vigevani Chair in Statistics. 
When, in addition, $\int_{(0,1]} x v(\mathrm{~d} x)<\infty$ then the Lévy process is an independent sum of a Brownian motion and a pure jump subordinator (nondecreasing Lévy process). In this case, $\varphi$ becomes

$$
\varphi(\alpha)=c \alpha+\frac{\sigma^{2}}{2} \alpha^{2}-\int_{(0, \infty)}\left(1-\mathrm{e}^{-\alpha x}\right) v(\mathrm{~d} x),
$$

where $c=\mu+\int_{(0, \infty)} x v(\mathrm{~d} x)$.

It is also well known that, for any Lévy process with no negative jumps and $\varphi^{\prime}(0)>0$, if we define $M=\sup _{t \geq 0} X_{t}$ then

$$
\mathrm{Ee}^{-\alpha M}=\frac{\alpha \varphi^{\prime}(0)}{\varphi(\alpha)},
$$

and that the limiting and stationary distributions of the (Markov) process $W_{t}=X_{t}+L_{t}$, where $L_{t}=-\inf _{0 \leq s \leq t} X_{s}$, is the distribution of $M$. We (and others) call the formula $\alpha \varphi^{\prime}(0) / \varphi(\alpha)$ the generalized Pollaczek-Khinchine (PK) formula. The reason for the name is that, for the special case of the M/G/1 queue, the underlying driving (Lévy) process is a compound Poisson process (a subordinator) minus $t$ for which the Laplace-Stieltjes transform of the limiting and stationary distributions of the workload process is the celebrated PK formula and may be found in virtually all basic queueing theory textbooks.

There are various textbooks where Lévy processes are discussed and where the above results may be either found directly or concluded from (see, e.g. [1], [3], [9], [14], and [13, pp. 19-34]). There are quite a few different proofs in the literature for the generalized PK formula, mostly via the application of the Wiener-Hopf factorization (see, e.g. [7] and [16]), weak convergence (see, e.g. [6] and [15]), and martingales (see, e.g. [8]), but this is not the scope here.

For some recent work on the distribution (rather than the Laplace-Stieltjes transform) of $\alpha$-stable Lévy processes, see [2], [10], [11], and the references therein. For the case with phasetype upward jumps (and general negative jumps), see [12]. For some other results, see also [5]. These papers also include an extensive list of references to texts and further related literature.

One is not required to be a Lévy process expert to read this paper and all the knowledge which is needed for what follows is covered above. In particular, this may easily be taught in any course where Lévy processes are touched upon.

\section{The case of an independent sum of a Brownian motion and a subordinator}

Let $X$ be a Lévy process which is an independent sum of a Brownian motion and a subordinator having a finite mean. In this case, as seen in the previous section, the (LaplaceStieltjes) exponent can be written in the form

$$
\varphi(\alpha)=c \alpha+\frac{\sigma^{2} \alpha^{2}}{2}-\int_{(0, \infty)}\left(1-\mathrm{e}^{-\alpha x}\right) v(\mathrm{~d} x),
$$

where $\bar{v} \equiv \int_{(0, \infty)} x v(\mathrm{~d} x)=\int_{0}^{\infty} v(x, \infty) \mathrm{d} x<c$. Defining

$$
F_{\mathrm{e}}(\alpha)=\int_{0}^{\infty} \mathrm{e}^{-\alpha x} \frac{\nu(x, \infty)}{\bar{v}} \mathrm{~d} x
$$

$\rho=\bar{v} / c<1$, and $\lambda=2 c / \sigma^{2}$, we observe that the exponent may be rewritten as

$$
\varphi(\alpha)=c \alpha\left(1+\frac{\alpha}{\lambda}-\rho F_{\mathrm{e}}(\alpha)\right)
$$


and, in particular, $\varphi^{\prime}(0)=c(1-\rho)$, so that the generalized PK formula has the form

$$
\frac{\alpha \varphi^{\prime}(0)}{\varphi(\alpha)}=\frac{1-\rho}{1+\alpha / \lambda-\rho F_{\mathrm{e}}(\alpha)}
$$

If $\sigma^{2}=0(\lambda=\infty)$ and $v(0, \infty)<\infty$, then we obtain the well-known (original) PK formula for the $\mathrm{M} / \mathrm{G} / 1$ queue. When $v(0, \infty)=\infty$, it is interesting to observe that exactly the same formula is valid without change, only $F_{\mathrm{e}}$ is then the stationary excess life distribution associated with the jumps of the subordinator and, unlike in the renewal process setting, its density approaches $\infty$ in the neighborhood of 0 . If the Lévy measure is 0 then $\rho=0$ and the formula becomes the Laplace-Stieltjes transform (LST) of an exponential distribution with rate $\lambda$. This is also well known to be the LST of the stationary distribution associated with a one-dimensional reflected Brownian motion with negative drift. The interesting discovery is that these two results can be unified. To see this, note that

$$
\frac{\alpha \varphi^{\prime}(0)}{\varphi(\alpha)}=\frac{\lambda}{\lambda+\alpha} \frac{1-\rho}{1-\rho F_{\mathrm{e}}(\alpha) \lambda /(\lambda+\alpha)}=\sum_{n=0}^{\infty}(1-\rho) \rho^{n} F_{\mathrm{e}}^{n}(\alpha)\left(\frac{\lambda}{\lambda+\alpha}\right)^{n+1},
$$

and so we have the following result.

Theorem 1. For a Lévy process with no negative jumps satisfying $\int_{(0,1]} x v(\mathrm{~d} x)<\infty$, $\varphi^{\prime}(0)>0$, and with the notation defined above, let $N \sim G(1-\rho)$ in the sense that $\mathrm{P}[N=$ $n]=(1-\rho) \rho^{n}$. Let $X_{0}, X_{1}, X_{2}, \ldots \sim \exp (\lambda)$ (equaling 0 for $\left.\lambda=\infty\right)$ and $Y_{1}, Y_{2}, \ldots \sim F_{\mathrm{e}}$, and assume that $N, X_{0}, X_{1}, \ldots$ and $Y_{1}, Y_{2}, \ldots$ are all independent. Then $\alpha \varphi^{\prime}(0) / \varphi(\alpha)$ is the LST of the random variable

$$
X_{0}+\sum_{n=1}^{N}\left(X_{n}+Y_{n}\right)
$$

where an empty sum is 0 .

When $\bar{v} \downarrow 0$, then we are left with $X_{0}$ as expected (the Brownian motion case) and, when $\sigma^{2} \downarrow 0$, we are left with $\sum_{n=1}^{N} Y_{n}$ also as expected (distributional form of the PK formula).

An interesting special case occurs when the jumps of the Lévy process have a phase-type distribution. Then the residual life also has a phase-type distribution, and, thus, also $X_{i}+Y_{i}$ and $X_{0}+\sum_{n=1}^{N}\left(X_{n}+Y_{n}\right)$. This is a special case of the results reported in [12], but with an easier proof and a far less complicated setup.

It is very easy to check that a converse also holds.

Theorem 2. Assume that $0<p \leq 1,0<\lambda \leq \infty$, and that $f$ is nonnegative and nonincreasing with $\int_{0}^{\infty} f(y) \mathrm{d} y=1$ (possibly with $f(x) \rightarrow \infty$ as $x \downarrow 0$ ), and define $F(x)=\int_{0}^{x} f(y) \mathrm{d} y$. Let $N \sim G(p), X_{0}, X_{1}, \ldots \sim \exp (\lambda)$ (equaling 0 for $\lambda=\infty$ ), and $Y_{1}, Y_{2}, \ldots \sim F$, where all random variables are independent. Then there exists a Lévy process which is an independent sum of a Brownian motion and a subordinator having a negative mean for which $\alpha \varphi^{\prime}(0) / \varphi(\alpha)$ is the LST of

$$
X_{0}+\sum_{n=1}^{N}\left(X_{n}+Y_{n}\right)
$$

where an empty sum is 0 . This Lévy process is unique up to a constant time scale. 
Proof. To see this, we can simply perform reverse engineering. First assume without loss of generality that $f$ is right continuous; otherwise, we take its right-continuous version which gives the same $F$. Let $v((x, \infty))=\beta f(x)$ for any constant $\beta>0$ and, thus, $v((a, b])=$ $\beta(f(a)-f(b))$, which uniquely characterizes $v$. Note that

$$
\int_{(0, \infty)} x v(\mathrm{~d} x)=\int_{0}^{\infty} v((x, \infty)) \mathrm{d} x=\int_{0}^{\infty} \beta f(x) \mathrm{d} x=\beta<\infty,
$$

as required. Recalling that $\rho=\bar{v} / c$ and that necessarily $p=1-\rho$, then since $\bar{v}=\beta$, we must set $c=\beta /(1-p)$. Now, if $\lambda=\infty$, we set $\sigma^{2}=0$; otherwise, from $\lambda=2 c / \sigma^{2}$ we set $\sigma^{2}=2 \beta / \lambda(1-p)$. Finally,

$$
\varphi^{\prime}(0)=c-\bar{v}=\frac{\beta}{1-p}-\beta>0,
$$

so that all requirements are met.

The fact that this Lévy process is unique up to a constant time scale is evident since if

$$
\frac{\alpha \varphi^{\prime}(0)}{\varphi(\alpha)}=\frac{\alpha \psi^{\prime}(0)}{\psi(\alpha)}
$$

then necessarily $\psi(\alpha)=\gamma \varphi(\alpha)$ for $\gamma=\psi^{\prime}(0) / \varphi^{\prime}(0)$, so that if $X$ is a Lévy process with exponent $\varphi$ and $Y$ with $\psi$, then $\left\{X_{\gamma t} \mid t \geq 0\right\}$ is distributed like $Y$.

\section{The general spectrally positive case}

The fact that $\alpha \varphi^{\prime}(0) / \varphi(\alpha)$ is the LST of some proper distribution for any Lévy process with no negative jumps and a negative mean is usually deduced indirectly from the fact that it must be the LST of either the supremum or the stationary LST of the reflected process. Alternatively, it can also be deduced from the fact that

$$
\frac{\varphi(\alpha)}{\alpha \varphi^{\prime}(0)}=1+c^{-1} \int_{0}^{\alpha}\left(\frac{\sigma^{2}}{2}+\int_{0}^{\infty} \mathrm{e}^{-\beta x} x v(x, \infty) \mathrm{d} x\right) \mathrm{d} \beta
$$

has a completely monotone derivative (see, e.g. the discussion above Proposition 8 of [4, pp. 728-729]). However, in our view another even more elementary and direct way of showing this is via Theorems 1 and 2 together with the following.

Observe that if $\int_{(0,1]} x v(\mathrm{~d} x)=\infty$ then the Lévy process is not a sum of a Brownian motion and a subordinator. In this case (since $\varphi^{\prime}(0)>0$ and, thus, $\int_{(1, \infty)} x v(\mathrm{~d} x)<\infty$ ), we recall from (1) that

$$
\varphi(\alpha)=\mu \alpha+\frac{\sigma^{2} \alpha^{2}}{2}+\int_{(0, \infty)}\left(\mathrm{e}^{-\alpha x}-1+\alpha x\right) \nu(\mathrm{d} x)
$$

and that $\varphi^{\prime}(0)=\mu$. Now, with

$$
\varphi_{\varepsilon}(\alpha)=\mu \alpha+\frac{\sigma^{2} \alpha^{2}}{2}+\int_{(\varepsilon, \infty)}\left(\mathrm{e}^{-\alpha x}-1+\alpha x\right) v(\mathrm{~d} x)
$$

for $\varepsilon>0$, we clearly have $\varphi_{\varepsilon}(\alpha) \rightarrow \varphi(\alpha)$ as $\varepsilon \downarrow 0, \varphi_{\varepsilon}^{\prime}(0)=\varphi^{\prime}(0)=\mu$ and, thus,

$$
\lim _{\varepsilon \downarrow 0} \frac{\alpha \varphi_{\varepsilon}^{\prime}(0)}{\varphi_{\varepsilon}(\alpha)}=\frac{\alpha \varphi^{\prime}(0)}{\varphi(\alpha)},
$$

and $\alpha \varphi^{\prime}(0) / \varphi(\alpha) \rightarrow 1$ as $\alpha \downarrow 0$. 
Now $\varphi_{\varepsilon}$ is the exponent of a Lévy process which is an independent sum of a Brownian motion and a compound Poisson process for which we have identified the distribution associated with $\alpha \varphi_{\varepsilon}^{\prime}(0) / \varphi_{\varepsilon}(\alpha)$ in Theorem 1. From this, the following result is immediate.

Theorem 3. A distribution has the generalized PK LST for some Lévy process with no negative jumps and a negative mean if and only if it belongs to the closure of the family of distributions defined in Theorem 2.

Finally, since a compound geometric distribution and an exponential distribution are both infinitely divisible, this also gives an elementary proof of the fact that $\alpha \varphi^{\prime}(0) / \varphi(\alpha)$ is the LST of an infinitely divisible distribution.

\section{References}

[1] Applebaum, D. (2009). Lévy Processes and Stochastic Calculus, 2nd edn. Cambridge University Press.

[2] Bernyk, V., Dalang, R. C. And Peskir, G. (2008). The law of the supremum of a stable Lévy process with no negative jumps. Ann. Prob. 36, 1777-1789.

[3] Bertoin, J. (1996). Lévy Processes. Cambridge University Press.

[4] Bingham, N. H. (1975). Fluctuation theory in continuous time. Adv. Appl. Prob. 7, 705-766.

[5] Chaumont, L. (2010). On the law of the supremum of Lévy processes. Preprint. Available at http://arxiv.org/ abs/1011.4151v1.

[6] CzystoŁowsKi, M. And SzCZOTKA, W. (2010). Queueing approximation of suprema of spectrally positive Lévy process. Queueing Systems 64, 305-323.

[7] Harrison, J. M. (1977). The supremum distribution of a Levy process with no negative jumps. Adv. Appl. Prob. 9, 417-422.

[8] Kella, O. And Whitt, W. (1992). Useful martingales for stochastic storage processes with Lévy input. J. Appl. Prob. 29, 396-403.

[9] Kyprianou, A. E. (2006). Introductory Lectures on Fluctuations of Lévy Processes with Applications. Springer, Berlin.

[10] Kwaśnicki, M., MaŁecki, J. and Ryznar, M. (2011). Suprema of Lévy processes. Preprint. Available at http://arxiv.org/abs/1103.0935v1.

[11] Michna, Z. (2011). Formula for the supremum distribution of a spectrally positive $\alpha$-stable Lévy process. Statist. Prob. Lett. 81, 231-235.

[12] MordECKI, E. (2002). The distribution of the maximum of the Lévy process with positive jumps of phase-type. Theory Stoch. Process. 8, 309-316.

[13] Protter, P. E. (2003). Stochastic Integration and Differential Equations, 2nd edn. Springer.

[14] Sato, K.-I. (1999). Lévy Processes and Infinitely Divisible Distributions. Cambridge University Press.

[15] Szczotka, W. AND WoyczYŃski, W. A. (2003). Distributions of suprema of Lévy processes via the heavy traffic invariance principle. Prob. Math. Statist. 23, 251-272.

[16] Zolotarev, V. M. (1964). The first passage time of a level and the behavior at infinity for a class of processes with independent increments. Theory Prob. Appl. 9, 653-662. 